\title{
Effect of Various Veneering Techniques on Bond Strength and Colour Stability of Zirconia / Veneering Ceramic after Hydrothermal Aging
}

\author{
Rafat Sasany ${ }^{1}$, Duygu Sarac ${ }^{2}$, Goknil Ergun Kunt ${ }^{3}$ \\ ${ }^{1}$ Department of Prosthodontics, Samsun, Turkey, ${ }^{2,3}$ Department of Prosthodontics, \\ University of Ondokuz Mayis, Samsun, Turkey.
}

\section{ABSTRACT}

\section{BACKGROUND}

The purpose of this in-vitro study was to compare the colour stability and bond strength of zirconia-based ceramic restorations after hydrothermal aging using conventional layering, heat-pressing, and multilayer veneering techniques.

\section{METHODS}

One hundred twenty specimens' core ( $15 \times 10 \times 0.7)$ was fabricated from A2-shade zirconia CAD-CAM blocks (IPS e.max ZirCAD). Specimens were divided into 4 groups for veneering $(\mathrm{N}=30)$ : [(layering group (L), IPS e.max ceram), (heat - pressing group (P), IPS press), and multilayer group, IPS e max Cad for 2 different types of cement (cemented with RelyX U200 (M1) and PANAVIA SA (M2))]. Aging was performed for $\left(5-55^{\circ} \mathrm{C}, 5000\right)$. Colour coordinates before and after aging were measured to calculate colour differences $\left(\Delta \mathrm{E}_{00}\right)$. The shear bond strength test was performed with a universal test unit. The data were analysed using the analysis of variance (ANOVA) and Tukey's Honest Significant Difference test (alpha $=0.05$ ).

\section{RESULTS}

Significant differences were found between the groups in bond strength $(\mathrm{P}<0.001)$. Group M1 had the highest bond strength while Group L had the lowest bond strength. In terms of $\Delta \mathrm{E}_{00}$ values, there were significant variations between the groups $(\mathrm{P}<$ 0.001). Group M1 had the highest colour stability, while Group M2 had the lowest colour stability.

\section{CONCLUSIONS}

The method of fabrication had an impact on the power of the bond between veneering ceramic and zirconia, as well as its colour stability. The restoration fabricated by a multilayer technique was cemented by resin cement (Rely X U200 Automix) which was found to be more resistant and colour stable.

\section{KEY WORDS}

Veneers, Heat-Press Technique, Bond Strength, Colour Different, Hydrothermal Aging.
Corresponding Author: Dr. Rafat Sasany, Ulugazi 19 Mayis Blv. No: 16, Sa Dent, Samsun, Turkey. E-mail: sasanyr@gmail.com

DOI: $10.14260 /$ jemds/2021/704

How to Cite This Article:

Sasany R, Sarac D, Kunt GE. Effect of various veneering techniques on bond strength and colour stability of zirconia / veneering ceramic after hydrothermal aging. J Evolution Med Dent Sci 2021;10(39):34743479, DOI: 10.14260/jemds/2021/704

Submission 16-05-2021, Peer Review 07-09-2020,

Acceptance 14-09-2020, Published 27-09-2021.

Copyright (C) 2021 Rafat Sasany et al. This is an open access article distributed under Creative Commons Attribution License [Attribution 4.0 International (CC BY 4.0)] 


\section{BACKGROUND}

The production of many dental ceramics based on CAD / CAM technology ${ }^{1}$ has resulted from an increase in aesthetic demand and long-term restoration prospects. For CAD / CAM restorations, dental ceramics are becoming increasingly popular. 2,3,4 particularly, yttria partially stabilized tetragonal zirconia polycrystalline ( $\mathrm{Y}$ - TZP), because of its metastatic phase transition, which can improve fracture durability and strength.4,5 Y - TZP is opaque and can be veneered with feldspathic ceramic to look like natural teeth. ${ }^{6,7}$ However, the major problems of these types of ceramic restorations are chipping and delamination. ${ }^{3}$

Zirconia blocks are chalk white with limited translucency. To improve colour match, zirconia cores can be veneered and coloured with feldspathic ceramic. The typical failure of a veneer restoration is known as chipping of the zirconia veneer interface. This fracture pattern is associated with different veneering techniques, repeated firing, inhomogeneous ceramic layers ${ }^{7}$, mismatch in the coefficient of thermal expansion (CTE). ${ }^{7-11}$

The veneering restoration has been layered according to the conventional fabrication process of the zirconia-ceramic technique. ${ }^{11}$ Recently heat-pressing techniques and CAD-on or multilayer techniques ${ }^{12,13}$ by CAD / CAM technology ${ }^{19}$ have advanced for fabricated veneer ceramic restorations. ${ }^{11}$ These techniques are advantageous than the layering technique because it offers high speed, accuracy, and colour stability. ${ }^{10-13}$ Furthermore, shrinkage-related issues are eliminated, as are the effects of future sintering procedures. ${ }^{11,14-16}$

The optical properties of zirconia do not have desired aesthetics. ${ }^{17,18}$ To improve the optical behaviour of zirconia, additive, and colouring techniques have been proposed.19 Colouring of ceramics or glazes is common due to the dispersion of mineral pigments formed from crystalline phases. As a result, these crystal levels require structural and chemical stability, but they do dissolve during firing at high temperatures above $1200^{\circ} \mathrm{C}$ and hydrothermal, colour stability pigments may change. ${ }^{19-24}$

On the other hand, white zirconia, retains its colour properties after accelerated aging protocols, whereas in case of coloured zirconia's, colour properties may be altered due to hydrothermal degradation, causing it to become darker, redder, more yellow, and more opaque.25-31

Previous studies have reported that the effect of thermocycling can occur as a result of the repetitive contraction and expansion stresses caused by the different thermal coefficients of the restorative materials, that reduces the bond strength of the restoration. ${ }^{24-29} \mathrm{Also}$, the properties in ceramic materials may be altered due to hydrothermal degradation and cause a change in colour stability. 25,26

Macrotensile and microtensile tests have been used to measure bond strength. ${ }^{28}$ The microtensile test has been preferred for offering a more uniform stress distribution at the bonded surface. ${ }^{28}$ The strength of the macro-SBS bond The test is simple and quick, and there is no need to prepare additional specimens after transplantation. However, macro -SBS has drawbacks, such as uneven stress distribution across the interface. ${ }^{31}$ Knowledge about the efficiency of the multilayer technique aided by the CAD / CAM technology bonded, and with the resin cement is limited.

\section{Objectives}

The purpose of this in-vitro study was to compare the colour stability and bond strength of zirconia-based ceramic restorations after hydrothermal aging using conventional layering, heat-pressing, and multilayer veneering techniques. The null hypothesis stated that different veneering techniques did not affect the colour stability and bond strength of zirconia-based ceramic restorations.

\section{METHODS}

The study was conducted in the Department of Prosthodontics, University of Ondokuz mayis, Samsun, Turkey. It was an experimental randomized control trial. Study period was from 17 Jan 2019 to 23 May 2019. The study was approved by the institutional Ethical board of the Department of Prosthodontics, Samsun, Turkey.

One hundred twenty specimens (18 x $12 \times 0.85 \mathrm{~mm})$ of zirconia blocks according to in $\sim 20 \%$ shrinkage was sliced from pre-sintered CAD - CAM blocks (IPS e.max ZirCAD) using a slow-speed diamond saw (ISOMET, Buehler Ltd, Lake Bluff, IL USA) under running water. The final measurement was (15 x $12 \times 0.7 \mathrm{~mm}$ ). A digital caliper was used to measure the thickness of each specimen (Model Absolute Digimatic Caliper; Mitutoyo Corp, Japan). Following sintering, all specimens were abraded with airborne particles (Korox 50, BEGO, and Bremen, Germany) for 15 seconds from a distance of approximately 10 $\mathrm{mm}$, using $50 \mu \mathrm{m}$ aluminum oxide (Al203) particles at $2.5 \mathrm{bar}$. The specimens were then ultrasonically cleaned in distilled water for 10 minutes before being air-dried.

The zirconia specimens were divided into 3 groups for various veneering techniques. layering technique group (L, N = 30), heat-press technique group ( $\mathrm{P}, \mathrm{N}=30)$, and multilayered technique Group ( $M, N=60)$. The colour for all veneer ceramics was A2 according to VITA - shade scala . A thin layer $(0.1 \mathrm{~mm})$ of ceramic liner (ZL1 IPS e.max Ceram ZirLiner; Ivoclar, Vivadent) was applied to the veneering surface of 60 zirconia specimens and sintered according to the manufacturer's instructions for groups $\mathrm{L}$ and $\mathrm{P}$.

In Group L, A vinylpolysiloxane putty mold (Elite HD +, Zhermack SpA, Badia Polesine, Italy) ( $8 \times 6 \times 1 \mathrm{~mm}$ ) was used, which was veneered using the conventional layering technique. Ceramic powder (A2 / TI1 IPS e.max Ceram) and the build-up liquid was mixed and applied to the hole on the ceramic liner's surface, which was $\sim 0.5 \mathrm{~mm}$ in thickness. The excess moisture was removed with absorbent paper to reduce porosity after the vibration for condensation, and the silicone mold was carefully removed. Then, the ceramic was sintered (Programat P310; Ivoclar, Vivadent, Schaan, Liechtenstein) at $750^{\circ} \mathrm{C}$ for $1 \mathrm{~min}$. To compensate for the peripheral shrinkage of the porcelain, the veneering ceramic was applied in two layers. After the second application of porcelain, the second firing was carried out in the same manner as the first.

In Group P, A vinylpolysiloxane putty mold was used to create thirty wax patterns $(8 \times 6 \times 1 \mathrm{~mm})$ on the ceramic liner sheet that were invested with a proprietary investment material (IPS PressVEST Speed Investment; Ivoclar, Vivadent) and hot-pressed with ceramic ingots (LT A2 IPS e.max Press Refill Ingots; Ivoclar, Vivadent) at $925{ }^{\circ} \mathrm{C}$ for 20 min under a 
pressure of 4.5 bar in the furnace (Empress EP500; Ivoclar, Vivadent).

For all specimens of $\mathrm{L}$ and $\mathrm{P}$ group the upper side of the veneering ceramics were ground with aluminum oxide papers (\#240,400,600). Finally, glaze (Initial Glaze Powder; GC) was applied and was fired for 12 minutes at $710{ }^{\circ} \mathrm{C}$ in a furnace (Programat P310; Ivoclar, Vivadent, Schaan, Liechtenstein).

In M1 and M2 Groups, sixty rectangular lithium disilicate ceramic (IPS e.max CAD Refill; Ivoclar, Vivadent) superstructure with a final dimension of $(8 \times 6 \times 1 \mathrm{~mm})$ were prepared by using a slow-speed diamond saw (ISOMET, Buehler Ltd, Lake Bluff, IL USA) and were sintered in a hightemperature sintering furnace (In Fire HTC Speed; Sirona) at $1581{ }^{\circ} \mathrm{C}$ for $86 \mathrm{~min}$ by the manufacturer's instructions. Then, the glazing procedure was performed.

The inner surfaces of the lithium disilicate ceramic superstructures were etched with $9.5 \%$ hydrofluoric acid (Porcelain Etchant Gel, Bisco, Schaumburg, USA) for $20 \mathrm{~s}$, then rinsed with water for $60 \mathrm{~s}$ and air-dried. After the etching procedure, a silane coupling agent (Pre-Hydrolyzed Silane Primer; Bisco, Schaumburg, USA) was applied to the treated surfaces and then dried. On the other hand, the inner surface of the 60 zirconia specimens which were roughened by the airborne particle abrasion, were treated with zirconium-oxide ceramic primer (Z-primer; Bisco, Schaumburg, USA).

In groups, M1 and M2 the ceramic superstructures were cemented with different resin cement (M1: RelyX U200 Automix; 3M ESPE M2: PANAVIA SA; Cement Plus Automix Kuraray, Japan). Resin cement was dispensed from the Automix syringe and the desired amount was applied directly onto the treated surface of the superstructures. The lower and upper structures were fixed under a manual dynamometer with a constant force of 50 Newtons for $10 \mathrm{~min}$ and were polymerized with a light-curing unit (ELIPAR S10, 3M ESPE, Seefeld, Germany) with an output of $1200 \mathrm{~mW} / \mathrm{cm}^{2}$ for $3 \mathrm{~s}$. After cleaning the excess resin cement carefully, the specimens were polymerized for $20 \mathrm{~s}$ on all surfaces.

Under a regular illuminant (D65) (Master TL-D Super 80 18W / 865 1SL; Philips), the colour coordinates of all specimens were determined using the CIELab colour scale on a neutral gray background 35 by making use of a spectroradiometer ${ }^{36}$ (SpectraScan PR - 704; Photo Research). A saturated sucrose solution with an approximate refractive index of 1.5 was used between the specimens and the background and the colour data were recorded (L1*, a1*, b1*). ${ }^{35}$

The aging process of all specimens was performed by thermocycling in a distilled water for 5000 cycles between $5^{\circ} \mathrm{C}$ and $55 \pm 2^{\circ} \mathrm{C}$ on a thermal cycler (Dentester Solubris Technica). The dwelling time of each temperature was $30 \mathrm{~s}$, and the transfer time was $10 \mathrm{~s}$.

The colour differences after thermal aging were calculated by using CIEDE2000 $\left(\Delta \mathrm{E}_{00}\right)$ colour difference formula. ${ }^{37}$

$$
\sqrt{\left(\frac{\Delta L^{\prime}}{K_{L} S_{L}}\right)^{2}+\left(\frac{\Delta C^{\prime}}{K_{C} S_{C}}\right)^{2}+\left(\frac{\Delta H^{\prime}}{K_{H} S_{H}}\right)^{2}+R_{T}\left(\frac{\Delta C^{\prime}}{K_{C} S_{C}}\right)\left(\frac{\Delta H^{\prime}}{K_{H} S_{H}}\right)}
$$

The CIEDE2000 colour difference formula's parametric factors were set to $1 .{ }^{37} \Delta \mathrm{E}_{00} \leq 1.30$ was considered as the perceptibility, and $\Delta \mathrm{E}_{00}>2.25$ as the acceptability threshold. ${ }^{38}$
Acrylic resin (3 x $3 \mathrm{~cm}$ ) (Meliodent; Kulzer, Hanau, Germany) was used to embed all the specimens. The macroSBS test was then performed on a universal testing machine (M500 - $25 \mathrm{KN}$; Testometric) at a crosshead speed of $1 \mathrm{~mm} /$ min until fracture occurred.

The failure mode of all specimens after the macro-SBS test was examined by using a field emission scanning electron microscopy (SEM; JSM-840A $6335 \mathrm{~F}$, Jeol, Japan) at a magnification of $\times 50$.

\section{Statistical Analysis}

The data were statistically analysed using IBM SPSS V23 (IBM Corp.). The mean SBS values were analysed by Variance (ANOVA) and sample t-test (for all tests).

\section{RESULTS}

One-way ANOVA indicated that veneering technique affected the bond strength and colour change within the ceramic veneer to zirconia $(\mathrm{P}<0.001)$ (Table 1 and 2). Two-way ANOVA indicated that hydrothermal aging affected the colour change within the ceramic veneer to zirconia $(\mathrm{P}<0.001)$ (Table 3). The higher bond strength values were obtained for Group M1 (26.45) and the lower bond strength values for L Group (22.59). No significant differences were found between the groups $\mathrm{L}$ and $\mathrm{P}$, also for the $\mathrm{M} 1$ and $\mathrm{M} 2$ Groups.

The lower $\Delta \mathrm{E}_{00}$ values were found for M1 Group (0.65) and the higher $\Delta \mathrm{E}_{00}$ values were found for Group $\mathrm{L}$ (1.35). No significant differences were found between the P, M1, and M2 Groups. The $\Delta \mathrm{E}_{00}$ values of all groups were below clinically acceptable thresholds $\left(\Delta \mathrm{E}_{00} \leq 2.25\right)$. The $\Delta \mathrm{E}_{00}$ values of Group M1, M2, and P were imperceptible and clinically acceptable $\left(\Delta \mathrm{E}_{00} \geq 1.30\right)$

The failure mode of all specimens after the macro-SBS test was adhesive failures.

\begin{tabular}{|cccc|}
\hline Group & Mean \pm SD & $\begin{array}{c}\text { Test } \\
\text { statistics }\end{array}$ & P \\
Group L & $20.59 \pm 1.11^{\mathrm{a}}$ & & \\
Group P & $24.98 \pm 0.90 \mathrm{a}$ & $\mathrm{T}=153.244$ & $<0.001$ \\
Group M1 & $26.45 \pm 1.25^{\mathrm{b}}$ & & \\
Group M2 & $25.52 \pm 0.85^{\mathrm{b}}$ & \\
\hline \multicolumn{4}{|r}{ Table 1. One -Way ANOVA for the Effect of } \\
Veneering Technique on Bond Strength & \\
\hline
\end{tabular}

Same superscript letters indicate no significant difference

\begin{tabular}{|cccc|}
\hline Group & Mean \pm SD & $\begin{array}{c}\text { Test } \\
\text { Statistics }\end{array}$ & P \\
Group L & $1.35 \pm 0.54^{\mathrm{a}}$ & & \\
Group P & $1.10 \pm 0.98^{\mathrm{b}}$ & $\mathrm{T}=42.461$ & $<0.001$ \\
Group M1 & $1 \pm 1.4^{\mathrm{b}}$ & & \\
Group M2 & $0.60 \pm 1.21^{\mathrm{b}}$ & \\
\hline \multicolumn{3}{r}{ Table 2. One-Way ANOVA for the Effect of } \\
Veneering Technique on DEoo
\end{tabular}

Same superscript letters indicated no significant difference

\begin{tabular}{|cccc|}
\hline Parameter & df & F & P \\
Veneering technique & 3 & 65.91 & $<0.001$ \\
Aging & 3 & 6.89 & $<0.001$ \\
Veneering techniques x aging & 3 & 13.59 & $<0.001$ \\
\hline Table 3. Two-Way ANOVA after Hydrothermal & \\
Aging on Colour Stability & & \\
\hline \multicolumn{4}{|c}{} \\
\hline
\end{tabular}




\section{DISCUSSION}

In the present study, because different veneering techniques had effects on the bond strength and colour change of ceramic veneer to zirconia after hydrothermal aging, the null hypotheses were rejected $(\mathrm{P}<0.001)$. In a previous study, it was recently reported that the heat-pressing group showed higher bond strength than the layering group, and this result was explained by the differences in the substructure and superstructure combination. ${ }^{15}$ Also, Lopez Molla et al. compared bond strength after layering and -pressing veneering ceramics to the zirconia core, and they stated that the heat-pressing group showed higher bond strength than layering group. ${ }^{30}$ In Group L and P, the thickness of the ceramic liner was $\sim 0.1 \mathrm{~mm}$, according to the manufacturer's instructions. Another research invested into the impact of the thickness of the ceramic liner on the bond strength of zirconia and veneering ceramic, concluding that $0.1 \mathrm{~mm}$ thickness of ceramic liner application decreased the bond strength when compared to $0.2 \mathrm{~mm}$ thickness of ceramic liner application group and to the control group which had no treatment. ${ }^{17}$ Also, Ereifej et al. ${ }^{39}$ reported that layering the veneering ceramic on zirconia cores resulted in lower bond strength due to the liner material's inadequate wettability on the zirconia surface.

In another study, the fracture resistance of all-ceramic first molar crowns with Y - TZP infrastructures was compared using three different veneering techniques (layering, press-on, and multi-layered). Multi-layered restorations made from CAD / CAM blocks also had significantly higher fracture strength values, according to the report. ${ }^{31}$

Previous studies reported that CAD / CAM veneers cemented to zirconia showed lower fracture strength than the layering technique. ${ }^{3,10}$ Additionally, in another study the bond strength between the zirconia core and veneers fabricated by different techniques, were compared. Furthermore, it was reported that the lower bond strength values were obtained with the CAD / CAM veneers cemented to zirconia specimens without any surface treatment when compared to the layering technique and the groups treated with different surface treatment and fused with fusion porcelain. ${ }^{15}$ The variations in the physical properties of the bi-layered and multi-layered ceramics under mechanical load were used to explain this result. The crack propagation and mechanical behaviour of the layered structure are influenced by interfacial properties and variations in the elastic modulus of the materials. ${ }^{12}$ The rigid zirconia's supportive impact on the brittle veneering ceramic may have been reduced due to the resin cement's lower elastic modulus. ${ }^{39}$ However, for the porcelain-based crowns, it is well known that using resin cement to blunt the defects of ceramic restorations can improve fracture resistance. ${ }^{40}$ Additionally, resin cement type can affect the bond strength of the two different surfaces or materials. There is no universal resin cement presented yet, which can be applied in all restorative procedures, therefore clinicians should know the applied resin cement properties, such as water sorption, bond strength, polymerization shrinkage, and application procedures, etc. For this reason, these properties were investigated by the previous study, and they can affect the bond strength of veneering ceramic to zirconia core. 41 Previous studies investigated the effect of various veneering techniques on the bond strengths of zirconia-based ceramic restorations. ${ }^{11,15,21,39}$
It has been reported that the multilayer technique, in which the zirconia core and the veneering ceramic were bonded using a fusion glass-ceramic, showed higher strength values than layering and heat-pressing techniques. ${ }^{12}$ Especially, previous studies have used a fusion glass-ceramic as a bonding agent for the multilayer technique. ${ }^{12,13}$

The number and size of flaws in a ceramic restoration are linked to the materials and fabrication process. The CAD-CAM veneering method was expected to have the fewest defects due to its straightforward process and low number of firings. ${ }^{21,41}$ In this study also the higher bond strength was obtained with Group M1 that had a minimal firing procedure. However, when the two multilayer groups' bond strength was evaluated, no significant difference was found between them. The bond strength of M1 (Rely X U200) and M2(Panavia SA) cement groups were $26.45 \pm 1.25 \mathrm{MPa}$ and $25.52 \pm 0.85 \mathrm{MPa}$ respectively. Therefore, this result showed that the selfadhesive resin cement used for bonding veneering ceramic to zirconia may affect the bond strength.

The colour stability of dental ceramic materials is also significant. Metal oxide pigments and a small amount of zirconia core materials are applied to the porcelain. Pigment sedimentation alters the material's optical properties, resulting in colour shifts and variations. These adjustments can make achieving the desired colour stability more difficult. $^{22}$ Previous studies in which the effects of thermocycling and accelerated aging on the colour of ceramic restorations were investigated, states that thermocycling affects the final colour of the restorations, similar to the results of the present study. ${ }^{24-27}$ The automatic transformation of the tetragonal to $\mathrm{ZrO} 2$ process to monoclinic caused by hydrothermal aging modified the optical and chemistry of grain boundaries. ${ }^{34}$ Additionally, the authors reported that the effect of pore population and pore size as low as $0.05 \%$ may decrease tolerance to LTD by facilitating the diffusion of water cause of light scattering in zirconia and colour stability. ${ }^{20}$

In the present study, after thermocycling, significant differences were found between the groups $(\mathrm{P}<.001)$. The lower $\Delta \mathrm{E}_{00}$ values were obtained with Group M1 $(0.65 \pm 1.21)$ and the higher $\Delta \mathrm{E} 00$ values were obtained with Group L $(1.35$ \pm 0.54 ). The colour and translucency of the substance changes as the sintering progresses. ${ }^{20}$ When the Y-TZP is fired under controlled conditions or sintering is done in a low-reaction setting, oxygen vacancies form. ${ }^{20}$

This difference may be because of the pores on the surface of the ceramic, which may decrease the amount of light returned.

\section{CONCLUSIONS}

Within the limitations of this in vitro study, the following conclusions were drawn -

1. The veneering technique affected the colour change and bond strength of veneering ceramic to zirconia after thermocycling.

2. Layering technique which requires multiple applications and firing cycles showed lower bond strength and colour stability values.

3. Multilayer technique groups, in which the two components of the restoration are bonded by a resin 
cement, showed higher bond strength and colour stability values.

Data sharing statement provided by the authors is available with the full text of this article at jemds.com.

Financial or other competing interests: None.

Disclosure forms provided by the authors are available with the full text of this article at jemds.com.

\section{REFERENCES}

[1] Guess PC, Schultheis S, Bonfante EA, et al. All-ceramic systems: laboratory and clinical performance. Dent Clin North Am 2011;55(2):333-52.

[2] Juntavee N, Sirisathit I. Marginal accuracy of computeraided design and computer-aided manufacturingfabricated full-arch zirconia restoration. Clin Cosmet Investig Dent 2018;10:9-17.

[3] Schmitter M, Mueller D, Rues S. In vitro chipping behavior of all-ceramic crowns with a zirconia framework and feldspathic veneering: comparison of CAD/CAMproduced veneer with manually layered veneer. J Oral Rehabil 2013;40(7):519-25.

[4] Miyazaki T, Nakamura T, Matsumura H, et al. Current status of zirconia restoration. J Prosthodont Res 2013;57(4):236-61.

[5] Sasany R, Ceylan G, Koca MF, et al. Effect of potassium nitrate and boric acid on the bond strength of veneering ceramics to zirconia. Eur J Prosthodont Restor Dent 2020;28(4):182-8.

[6] Ahmad S, Ahed A, Radi M. Zirconia-based restorations: literature review. Int J Med Prof 2017;3(2):253-60.

[7] Tanış CM, Kılıçarslan MA, Bellaz IB. In vitro evaluation of bond strength between the zirconia core and CAD/CAM produced veneers. J Prosthodont 2020;29(1):56-61.

[8] Guess PC, Bonfante EA, Silva NR, et al. Effect of core design and veneering technique on damage and reliability of YTZP-supported crowns. Dent Mater 2013;29(3):307-16.

[9] Lima JMC, O'Souza AC, Anami LC, et al. Effects of thickness, processing technique and cooling rate protocol on the flexural strength of a bilayer ceramic system. Dent Mater 2013;29(10):1063-72.

[10] Al-Wahadni A, Shahin A, Kurtz KS. Veneered zirconiabased restorations fracture resistance analysis. J Prosthodont 2018;27(7):651-8.

[11] Choi YS, Kim SH, Lee JB, et al. In vitro evaluation of fracture strength of zirconia restoration veneered with various ceramic materials. J Adv Prosthodont 2012;4(3):162-9.

[12] Alessandretti R, Ribeiro R, Borba M, et al. Fracture load and failure mode of CAD-on ceramic structures. Braz Dent J 2019;30(4):380-4.

[13] Wiedhahn K. The impression-free cerec multilayer bridge with the CAD-on method. Int J Comput Dent 2011;14(1):33-45.

[14] Sim JY, Lee WS, Kim JH, et al. Evaluation of shear bond strength of veneering ceramics and zirconia fabricated by the digital veneering method. J Prosthodont Res 2016;60(2):106-13.

[15] Ishibe M, Raigrodski AJ, Flinn BD, et al. Shear bond strengths of pressed and layered veneering ceramics to high-noble alloy and zirconia cores. J Prosthet Dent 2011;106(1):29-37.

[16] Stawarczyk B, Özcan M, Roos M, et al. Load-bearing capacity and failure types of anterior zirconia crown veneered with overpressing and layering techniques. Dent Mater 2011;27(10):1045-53.

[17] Sasany R, Saraç D, Özcan M. Effect of different liner techniques and argon plasma treatment of zirconia base on the adhesion and colour change of veneering ceramic. J Adhesion Sci Technol 2021:1-14.

[18] Volpato CAM, Cesar FP, Bottino MA. Influence of accelerated aging on the colour stability of dental zirconia. J Esthet Restor Dent 2016;28(5):304-12.

[19] Nam JY, Park MG. Effects of aqueous and acid-based colouring liquids on the hardness of zirconia restorations. J Prosthet Dent 2017;117(5):662-8.

[20] Alghazzawi TF. The effect of extended aging on the optical properties of different zirconia materials. J Prosthodont Res 2017;61(3):305-14.

[21] Fathi A, Farzin M, Giti R, etal. Effects of a number of firings and veneer thickness on the colour and translucency of 2 different zirconia-based ceramic systems. J Prosthet Dent 2019;122(6):565.e1-7.

[22] Yamashita I, Tsukuba K. Light scattering by residual pores in transparent zirconia ceramics. J Ceram Soc Jpn 2011;119(1386):133-5.

[23] Lee YK, Cha HS, Ahn JS. Layered colour of all-ceramic core and veneer ceramics. J Prosthet Dent 2007;97(5):279-86.

[24] Vichi A, Sedda M, Fonzar RF, et al. Comparison of contrast ratio, translucency parameter and flexural strength of traditional and augmented translucency zirconia for CEREC CAD/CAM system. J Esthet Restor Dent 2016;28(Suppl 1):32-9.

[25] Haralur SB, Alqahtani NRS, Mujayri FA. Effect of hydrothermal aging and beverages on colour stability of lithium disilicate and zirconia based ceramics. Medicina (Kaunas) 2019;55(11):749.

[26] Hamza TA, Alameldin AA, Elkouedi AY, et al. Effect of artificial accelerating aging on surface roughness and colour stability of different ceramic restoration. Stomatalogical Dis Sci 2017;1:8-13.

[27] Vasiliu RD, Porojan SD, Birdeanu MI, et al. Effect of thermocycling, surface treatments and microstructure on the optical properties and roughness of CAD-CAM and heat-pressed glass ceramics. Materials (Basel) 2020;13(2):381.

[28] Zhang F, Inokoshi M, Batuk M, et al. Strength, toughness and aging stability of highly-translucent Y-TZP ceramics for dental restorations. Dent Mater 2016;32(12):e327-37.

[29] Swain MV. Impact of oral fluids on dental ceramics: what is the clinical relevance? Dent Mater 2014;30(1):33-42.

[30] Van Meerbeek B, Peumans M, Poitevin A, et al. Relationship between bond-strength tests and clinical outcomes. Dent Mater 2010;26(2):100-21.

[31] Lopez-Molla MV, Martinez-Gonzalez MA, Manes-Ferrer JF, et al. Bond strength evaluation of the veneering-core ceramics bonds. Med Oral Patol Oral Cir Bucal 2010;15(6):e919-23.

[32] Ereifej N, Rodrigues FP, Silikas N. Experimental and FE shear bond strength at core/veneer interfaces in bilayered ceramics. Dent Mater 2011;27(6):590-97. 
[33] Van Meerbeek B, Peumans M, Poitevin A, et al. Relationship between bond-strength tests and clinical outcomes. Dent Mater 2010;26(2):100-21.

[34] Sasany R, Ergun-Kunt G, Yilmaz B. Effect of mouth rinses on optical properties of CAD-CAM materials used for laminate veneers and crowns. J Esthet Restor Dent 2021;33(4):648-53.

[35] Pelino JEP, Passero A, Martin AA, et al. In vitro effects of alcohol-containing mouthwashes on human enamel and restorative materials. Braz Oral Res 2018;32:e25.

[36] Spink LS, Rungruanganut P, Megremis S, et al. Comparison of anabsolute and surrogate measure of relative translucency in dental ceramics. Dent Mater 2013;29(6):702-7.

[37] Luo MR, Cui G, Rigg B. The development of the CIE 2000 colour-difference formula: CIEDE2000. Colour Res Application 2001;26(5):340-50.
[38] Commission Internationale de I'Eclairage Colourimetry. $4^{\text {th }}$ edn. Vienna: Commission Internationale de I'Eclairage 2019: p. 111.

[39] Paravina RD, Ghinea R, Herrera LJ, Bona AD, Igiel C, Linninger $\mathrm{M}$ et al. Colour difference thresholds in dentistry. J Esthet Restor Dent 2015;1:S1-9.

[40] Kanat-Ertürk B, Çömlekoğlu EM, Dündar-Çömlekoğlu M, et al. Effect of veneering methods on zirconia frameworkveneer ceramic adhesion and fracture resistance of single crowns. J Prosthodont 2015;24(8):620-8.

[41] Kaimal A, Ramdev P, Shruthi CS. Evaluation of the effect of zirconia surface treatment, using plasma of Argon and silane, on the shear bond strength of two composite resin cement. J Clin Diagn Res 2017;11(8):ZC39-43.

[42] Da Silva NR, De Paula Rodrigues M, Bicalho AA, et al. Effect of resin cement mixing and insertion method into the root canal on cement porosity and fiberglass post bond strength. J Adhes Dent 2019;21(1):37-46. 